\title{
AVALIAÇÃO DA EFICIÊNCIA DE UM SISTEMA DE LAGOAS DE ESTABILIZAÇÃO DE ESGOTOS NA REMOÇÃO DE OVOS DE HELMINTOS: PROPOSTA PARA A DETERMINAÇÃO DO PERCENTUAL DE RECUPERAÇÃO DE OVOS DE HELMINTOS ATRAVÉS DO MÉTODO DE SCHWARTZBROD
}

\author{
Wilma Maria Coelho , Dulcinéa Maria Barbosa Campos **
}

\begin{abstract}
RESUMO
Este estudo teve como objetivo verificar a capacidade de retenção de ovos de helmintos num sistema formado por lagoas de estabilização de esgotos e para isso foram testados e avaliados três métodos (EXTRABES, Leeds I e o método A recomendado pela Organizaçăo Mundial de Saúde, descrito por Schwartzbrod (WHO,1989).

Por não existirem dados disponíveis sobre o percentual de recuperaçăo de ovos de helmintos pelo método de Schwartzbrod, foi proposto um procedimento para determinar tal percentual. Os ensaios foram realizados em amostras com volume de sólidos sedimentáveis variando de $1,0 \mathrm{ml}$ a $20,0 \mathrm{ml}$ resultando percentuais de recuperação de ovos de helmintos entre $18 \%$ e $3,3 \%$. Observou-se que o número de ovos de helmintos encontrados através deste método foi maior em amostras que apresentam menor quantidade de sólidos sedimentáveis. Estes resultados demonstram que ao realizar o exame parasitológico do esgoto é necessário determinar previamente a quantidade de sólidos sedimentáveis na amostra para que ao final do exame seja possível aplicar os percentuais de recuperaçăo correspondentes aos valores de sólidos encontrados na amostra original.

Comparando os resultados obtidos, observou-se que o método de Schwartzbrod apresentou eficiência de recuperaçăo de ovos de helmintos 1,5 vezes maior que o método EXTRABES e 7,8 vezes maior que o método Leeds I.

$\mathrm{Na}$ remoção de ovos de helmintos, no sistema de lagoas de estabilizaçấo de esgotos, verificou-se que no efluente da lagoa primária $16,6 \%$ das amostras apresentaram-se fora dos padrōes recomendados pela Organizaçăo Mundial de Saúde para reutilizaçđ̃o na agricultura.
\end{abstract}

\footnotetext{
* Aluna do curso de especialização do Departamento de Parasitologia do IPTESP-UFGo

-* Professora Titular do Departamento de Parasitologia do IPTESP-UFGo

Recebido 25/06/96. Revisto em 10/09/96. Aceito em 28/10/96.
} 
COELHO, W.M.; CAMPOS, D.M.B. Avaliação da eficiência de um sistema de lagoas de estabilização de esgotos na remoção de ovos de helmintos: proposta para a determinação do percentual de recuperaço de ove. Trop.25 ( 1 ): 61-72, jan/jun. 1996

No efluente final todas as amostras examinadas apresentaram valores negativos, concluindose que, no período estudado, em termos de critérios parasitológicos a obedecer, o efluente final do sistema poderia ser reaproveitado em irrigaçð̌es restritas, conforme recomendaçסes da Organização sistema poderia ser reaproveitad
Mundial de Saúde (WHO, 1989).

UNITERMOS: Efluentes; ovos de helmintos; reutilização; lagoas de estabilização de esgoto.

\section{INTRODUÇÃo}

Atualmente, uma das maiores preocupações no saneamento básico tem sido o destino final e a possível reutilização dos efluentes das estações de tratamento de águas residuárias, na agricultura e aquacultura, principalmente em locais onde há baixos índices de pluviosidade e especialmente onde os recursos hídricos são escassos (Marecos do Monte, 1993).

Alguns estudos epidemiológicos têm indicado que algumas doenças podem estar associadas à reutilização de águas residuárias parcialmente tratadas e sem qualquer tipo de tratamento (Mara \& Cairncross, 1990). Além das doenças de origem virótica e bacteriana, várias doenças parasitárias são provocadas pela presença de estágios larvares dos helmintos e cistos de protozoários (Pawlowski, 1984).

Devido a ampla ocorrência de enteroparasitoses na população humana e animal, e a resistência apresentada pelos ovos dos helmintos (WHO, 1991) a pesquisa desses parasitas em amostras de águas residuárias a serem reutilizadas, constitui uma recomendação importante em estudos e programas de avaliação e monitoramento da qualidade ambiental, que visam minimizar a disseminação destes ovos no meio ambiente.

Os ovos de helmintos têm interesse, no contexto deste estudo, consoante às atividades de reutilização de águas tratadas em rega de culturas agrícolas, pelo fato de alguns (ex. os nematodas) possuirem um período de latência no solo antes de atingirem o hospedeiro vertebrado (Pawlowski, 1984). Dessa forma, a importância de estudá-los está na relação direta com o tipo de infecção que causam, seja pela ingestão de ovos ou penetração de larvas filarióides na pele. Por estes motivos é de todo o interesse a avaliação da qualidade das águas de rega.

Segundo a Organização Mundial de Saúde (WHO, 1989) um efluente para ser reutilizado na regade cereais, forragens, pastos e árvores frutíferas não deve
COELHO, W.M.; CAMPOS, D.M.B. Avaliaçăo da eficiencia de um sistema de lagoas de estabilizaçł ło de esgotos na remoção de ovos de helmintos: proposta para a determinaçấo do percentual de recuperação de ovos de helmintos através do método "OMS". Rev. Pat. Trop.25 ( 1 ): 61-72, jan./jun.
1996

conter mais que 1 ovo de nematoda por litro, condição essencial para a proteção dos trabalhadores na agricultura e dos consumidores de vegetais.

Neste sentido, através do presente trabalho propôs verificar a eficiência do sistema de tratamento de esgotos domésticos, da cidade de Inhumas-Goiás, quanto à remoção de ovos de helmintos. O objetivo foi observar se o tratamento oferecia condições de reaproveitamento de seus efluentes na rega, segundo critérios parasitológicos recomendados pela OMS. Para tanto foram testados três métodos: EXTRABES, Leeds I e o método de Schwartzbrod, e ainda realizados ensaios que resultaram numa proposta para a determinação do percentual de recuperação de ovos de helmintos deste último método.

\section{MATERIAIS E MÉTODOS}

\section{Características do sistema de lagoas de estabilização}

O sistema escolhido para realizar os ensaios do presente estudo, trata esgotos domésticos da cidade de Inhumas-Goiás e está situado a $35 \mathrm{~km}$ a noroeste da capital do Estado, sendo constituído por 2 lagoas de estabilização em série, Fig. 1. Segundo o memorial descritivo do projeto da empresa concessionária de água e esgoto, Saneamento de Goiás S/A, as lagoas estão dimensionadas como facultativas, apresentando áreas úteis de $31430,38 \mathrm{~m}^{2}$ e profundidade de $1,30 \mathrm{~m}$ (1 $1^{\mathrm{a}}$ lagoa) e 31 $016,38 \mathrm{~m}^{2}$ ( $2^{\mathrm{a}}$ lagoa), à mesma profundidade da anterior, cujos períodos de retençåo, em projeto, equivalem a 38 e 37,5 dias, respectivamente (Crispim, 1992). Com o assoreamento ocorrido nos últimos anos e falta de retirada do lodo nas lagoas acredita-se que este tempo de retenção tenha diminuído significativamente, não devendo ultrapassar 20 dias.

$\mathrm{O}$ esgoto afluente (bruto) passa por uma grade de retenção dos sólidos grosseiros, por uma caixa de areia e segue em direção a primeira lagoa imediatamente após o medidor Parshall.

O efluente final é lançado no rio Meia Ponte, aproximadamente, a $50 \mathrm{~km}$ à montante da captação de água para o abastecimento de parte da capital do Estado. A vazão média anual deste manancial é de $6,5 \mathrm{~m}^{3} / \mathrm{s}$ (Siqueira, 1996). 
COELHO, W.M.; CAMPOS, D.M.B. Avaliação da eficiência de um sistema de lagoas de estabilização de esgotos na remoção de ovos de helmintos: proposta para a determinaçăo do percentual de covs". Rev. Pat. Trop.25 ( 1 ): 61-72, jan.jun. recupe 1996

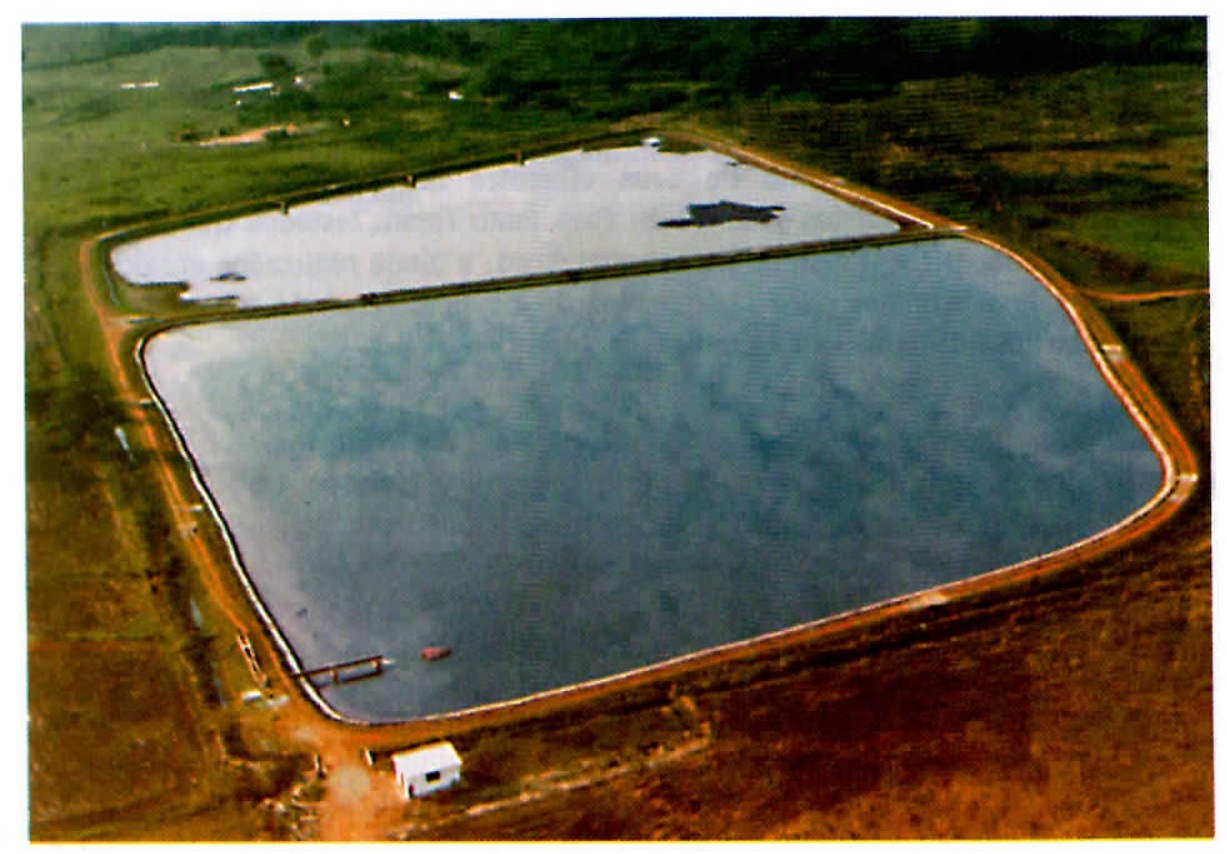

Fig. 1.Vista aérea das lagoas de estabilização de esgoto de Inhumas

\section{Coleta de amostras}

Para avaliação do sistema e seleção dos métodos foram colhidas amostras simples, na chegada do esgoto bruto, no efluente da $1^{\mathrm{a}}$ lagoa e no efluente final $\left(2^{\mathrm{a}}\right.$ lagoa), cujos volumes do líquido colhido variaram conforme as recomendações de cada método.

As amostras de esgoto bruto foram colhidas na calha Parshall e as amostras tratadas a aproximadamente 20 centímetros abaixo da superfície, no período da manhã entre 9 e 10 horas sendo transportadas, imediatamente, ao laboratório sem uso de solução preservante. A técnica para a coleta de amostras obedeceu às recomendações da Associação Americana de Saúde Pública (APHA, 1989).
COELHO, W.M.; CAMPOS, D.M.B. Avaliação da eficiência de um sistema de lagoas de estabilização de esgotos na remoção de ovos de helmintos: proposta para a determinação do percentual de recuperaçăo de ovos de helmintos através do método "OMS". Rev. Pat. Trop.25 ( 1 ): 61-72, jan./jun. 1996

Quando não foi possível analisar as amostras logo após colhidas, estas foram preservadas utilizando-se $10 \mathrm{ml}$ de formol comercial puro para cada litro e mantidas sob refrigeração a $4^{\circ} \mathrm{C}$.

O período da amostragem decorreu de Fevereiro a Julho de 1994, quinzenalmente, efetuando-se 12 coletas de amostras em cada ponto do sistema.

\section{Métodos testados}

Para a detecção dos ovos de helmintos foram aplicados 3 métodos (EXTRABES, Leeds I e o método de Schwartzbrod), em amostras de esgoto tratado e sem tratamento (esgoto bruto).

Método EXTRABES (apud Ayres et al, 1989): Este método foi empregado em amostras de esgoto sem tratamento e tratado. Para o exame do esgoto bruto foram colhidos $500 \mathrm{ml}$ de amostra conforme recomendação original do método. Nas amostras tratadas o volume inicial foi aumentado de 1 para 5 litros. Após sucessivas centrifugações da amostra foram examinados $0,2 \mathrm{ml}$ do sedimento de cada amostra. Para o cálculo final da amostra foi efetuada uma regra de três simples, onde sobre o valor encontrado foi aplicado um percentual de recuperação do método igual a $11 \%$.

Leeds I (Ayres et al, 1989): Conforme recomenda o método, este foi empregado somente a amostras sem tratamento, cujo volume inicial de 1 litro foi reduzido após várias centrifugações. Os ovos foram separados do sedimento através de solução de sulfato de magnésio saturado (densidade 1,300). As lâminas contendo os ovos foram observadas num período máximo de 30 minutos a fim de evitar a cristalização da amostra pela solução salina. De maneira análoga ao método anterior, sobre o valor encontrado efetuou-se uma regra de três simples considerando-se $24 \%$ o percentual de recuperação de ovos para o método Leeds I.

Método de Schwartzbrod (WHO, 1989): O volume para as amostras sem tratamento correspondeu a 1 litro e para as amostras tratadas o volume inicial foi aumehitađo de 1 para 10 litros (Ayres et al, 1991). Após sucessivas centrifugações o sedimento foi tratado com solução tampão aceto-acética $(\mathrm{pH} \mathrm{4,5)}$ e éter para a separação do material gorduroso dos ovos de helmintos. Em seguida estes foram ressuspensos por uma solução de sulfato de zinco (densidade 1,180). Registrou-se o número de ovos contados em câmara de Sedgwick-Rafter e a este valor aplicou-se o percentual de recuperação de ovos de helmintos em relação ao volume de sólidos sedimentáveis.

Por não ter conhecimento de dados publicados propôs-se um estudo experimental para avaliação da taxa de recuperação de ovos de helmintos pelo método de Schwartzbrod, por ser um dado importante e necessário para se conhecer a eficiência do método. 
COELHO, W.M.; CAMPOS, D.M.B. Avaliaçăo da eficiencia de um sistema de lagoas de estabilizaçăo de esgotos na remoção de ovos de helmintos: proposta para a determinaçăo do percentual de recuperação de ovos de helmintos através do método "OMS". Rev. Pat. Trop.25 ( 1 ): 61-72, jan./jun. 1996

\section{Análise estatística}

$\mathrm{Na}$ análise estatística foi usado um teste de diferenças de médias cuja segurança pode ser declarada através de um intervalo de confiança (IC) de $95 \%$, adotando-se uma distribuição t de Student. $O$ procedimento estatístico adotado para a comparação dos métodos foi a análise de duas amostras, onde estudou-se os três métodos, tomados dois a dois, em que o método de referência foi o método de Schwartzbrod. Foram calculados a média, o desvio padrão e o coeficiente de variação de cada amostra.

\section{Determinação da taxa de recuperação de ovos de helmintos pelo método de} Schwartzbrod

Com a finalidade de avaliar a taxa de recuperação de ovos de helmintos pelo método de Schwartzbrod foram efetuadas as seguintes etapas:

Em amostras de esgoto, escolhidas ao acaso, determinou-se previamente o volume dos sólidos sedimentáveis em cones de Imhoff por 1 hora (APHA, 1989), para saber se a quantidade de sedimento presente na amostra interferiria na recuperação de ovos.

Uma série de 10 amostras, cada amostra contendo 1 litro de esgoto foi contaminada com 1100 ovos de Ascaris suum preservados em solução de cloreto de sódio $0,85 \%$ e $\mathrm{H}_{2} \mathrm{SO} 40,1 \mathrm{~N}$ de concentração conhecida ( 1100 ovos/250 $\mu \mathrm{l}$ ).

Nos testes seguintes as quantidades de sólidos foram aumentadas, gradativamente e aleatoriamente com valores correspondendo a: $1 \mathrm{ml} / \mathrm{l} ; 2,5 \mathrm{ml} / \mathrm{l} ; 3,5$ $\mathrm{ml} / \mathrm{l} ; 5,0 \mathrm{ml} / \mathrm{l} ; 8,0 \mathrm{ml} / \mathrm{l} ; 10,0 \mathrm{ml} / \mathrm{l} ; 15,0 \mathrm{ml} / \mathrm{l} ; 20,0 \mathrm{ml} / \mathrm{l}$.

\section{RESULTADOS E DISCUSSÃo}

Os percentuais de recuperação de ovos de helmintos através do método de Schwartzbrod em amostras contaminadas com ovos de Ascaris suum encontram-se expressos na Tabela I.

Entre os testes realizados para a escolha da melhor função ajustante, a exponencial foi a que melhor se adequou aos dados obtidos. Observando-se a Tabela I e Fig. 2 verifica-se que o grau de recuperação de ovos de helmintos diminui à medida em que se aumenta o valor de sólidos na amostra.
COELHO, W.M.; CAMPOS, D.M.B. Avaliação da eficiência de um sistema de lagoas de estabilização de esgotos na remoção de ovos de helmintos: proposta para a determinação do percentual de recuperação de ovos de helmintos através do método "OMS". Rev. Pat. Trop.25 (1 ): 61-72, jan/jun. 1996

Tabela I. Determinação do percentual de recuperação de ovos de helmintos através do método de Schwartzbrod em relação ao volume de sólidos sedimentáveis (ss).

\begin{tabular}{cc}
\hline ss (ml) & $\%$ \\
\hline 1,0 & 18,0 \\
2,5 & 14,4 \\
3,5 & 14,0 \\
5,0 & 12,6 \\
8,0 & 12,4 \\
10,0 & 10,0 \\
15,0 & 6,6 \\
20,0 & 3,3 \\
\hline
\end{tabular}

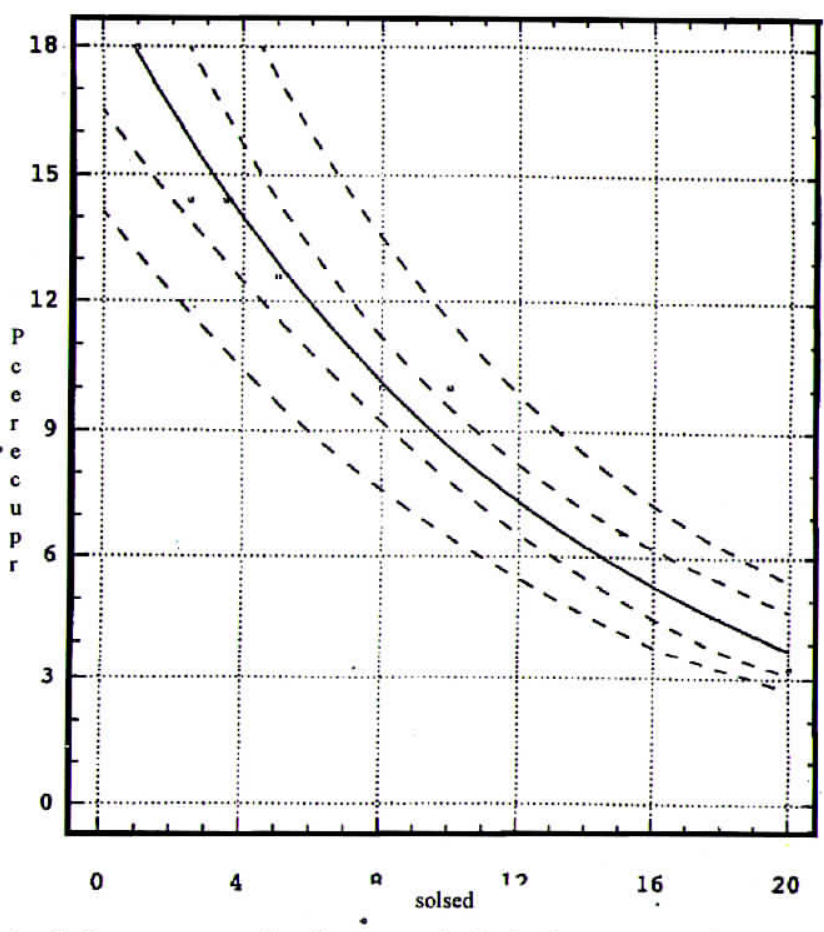

Fig. 2. Percentual de recuperação de ovos de helmintos em relação ao volume de sólidos na amostra 
COELHO, W.M.; CAMPOS, D.M.B. Avaliação da eficiência de um sistema de lagoas de estabilização de esgotos na remoção de ovos de helmintos: proposta para a determinação do percentual de recuperação de ovos de helmintos através do método "OMS". Rev. Pat. Trop.25 ( 1 ): 61-72, jan./jun. \begin{tabular}{l} 
recupe \\
1996 \\
\hline
\end{tabular}

\section{Comportamento do sistema}

Nas amostras de esgoto bruto foram encontrados ovos de nematoda (ancilostomídeos, Ascaris lumbricoides, Enterobius vermicularis, Trichuris trichiura) e de cestodas (Hymenolepis nana, Hymenolepis diminuta, Taenia sp).

Analisando-se as Tabela II e III verifica-se que através do método de Schwartzbrod recuperou-se um maior número de ovos de helmintos. Foi significativo o percentual de $66,7 \%$ de recuperação de ovos de helmintos pelo método Schwartzbrod em relação aos demais, com percentuais de $7,6 \%$ e $25,7 \%$ para o método de Leeds I e EXTRABES, respectivamente.

Tabela II. Número de ovos de helmintos recuperados em amostras de esgoto bruto (ovos/l)

\begin{tabular}{lrcr}
\hline & \multicolumn{3}{c}{ MÉTODO } \\
\cline { 2 - 4 } & Leeds I & Schwartzbrod & EXTRABES \\
\hline Ascaris lumbricoides & $\mathbf{7 8 2}$ & 7946 & 2000 \\
Ancilostomideos & 317 & 2474 & 1200 \\
Hymenolepis diminuta & 196 & 1185 & 800 \\
Hymenolepis nana & 71 & 273 & 400 \\
Trichuris trichiura & 04 & 202 & 220 \\
Enterobius vermicularis & 37 & 386 & 0 \\
Taenia sp. & 17 & 44 & 200 \\
Total de ovos/l por método & $\mathbf{1 4 2 4}$ & $\mathbf{1 2 5 1 0}$ & $\mathbf{4 8 2 0}$ \\
\hline
\end{tabular}

Tabela III. Percentual de ovos de helmintos recuperados por método em amostras de esgoto bruto

\begin{tabular}{lccc}
\hline & \multicolumn{3}{c}{ MÉTODO } \\
\cline { 2 - 4 } & Leeds I & Schwartzbrod & EXTRABES \\
\hline Ascaris lumbricoides & 7,3 & 74,1 & 18,6 \\
Ancilostomideos & 8,0 & 61,9 & 30,1 \\
Hymenolepis diminuta & 9,0 & 54,3 & 36,7 \\
Hymenolepis nana & 9,5 & 36,7 & 53,8 \\
Trichuris trichiura & 1,9 & 47,4 & 51,7 \\
Enterobius vermicularis & 8,7 & 91,3 & 0 \\
Taenia sp. & 6,5 & 16,9 & 76,6 \\
\% Total & $\mathbf{7 , 6}$ & $\mathbf{6 6 , 7}$ & $\mathbf{2 5 , 7}$ \\
\hline
\end{tabular}

COELHO, W.M.; CAMPOS, D.MB Avaliaçđo da eficiência de um sistema de lagoas de estabilização de esgotos na remoção de ovos de helmintos: proposta para a determinação do percentual de recuperação de ovos de helmintos através do método "OMS". Rev. Pat. Trop.25 ( 1 ): 61-72, jan./jun. 1996

Através da tabela IV observa-se o Coeficiente de Variação (CV) de Pearson em que a dispersão relativa dos dados, no método de Schwartzbrod, foi significativamente menor $(31,4 \%)$ que nos demais métodos. Estes dados traduziram uma maior homogeneidade de resultados e consequentemente, uma menor variabilidade do método na recuperação de ovos de helmintos.

A análise estatística das médias de ovos recolhidos demonstrou que o método de Schwartzbrod apresentou eficiência 1,5 vezes maior que o método EXTRABES e 7,8 vezes maior que o método de Leeds I.

Tabela IV. Análise estatística dos métodos aplicados às amostras de esgoto bruto

\begin{tabular}{lccc}
\hline \multicolumn{1}{c}{ Parâmetros } & Schwartzbrod & Leeds I & EXTRABES \\
\hline n. de amostras & 12 & 12 & 12 \\
média de ovos recolhidos & 1042 & 118 & 415 \\
desvio padrão & 327 & $\mathbf{5 9}$ & $\mathbf{2 4 0}$ \\
coeficiente de variação & $\mathbf{3 1 , 4 \%}$ & $49,9 \%$ & $\mathbf{5 7 , 8 \%}$ \\
\hline
\end{tabular}

A Organização Mundial de Saúde (WHO, 1989) considera que os ovos de helmintos particularmente os de nematoda oferecem um potencial de risco à saúde humana quando da reutilização de efluentes na irrigação. Os estudos de Shuval et al (apud OMS, 1989) atribuem maior importância a infecção por nematodas dado a forma passiva por ingestão de ovos ou ativa por penetração de larvas na pele.

No presente trabalho ao observar as Tabelas II e III constata-se a presença de ovos de Taenia sp, em amostras de esgoto bruto.

Alerta-se para a importância do complexo teníase-cisticercose em saúde pública em diversos países do mundo.

Vale lembrar que os ovos de Taenia sp são infectantes para os hospedeiros susceptíveis bovinos, suínos e humanos logo após serem eliminados não necessitando de um período de latência no solo. Shuval et al (apud OMS, 1989) alerta para a possibilidade de infecção com "Cysticercus bovis" (larva da Taenia saginata) quando o rebanho bovino se alimenta de pastos irrigados com esgoto bruto.

Na década de 70, em partes da África o índice de cisticercose animal era tão elevado ao ponto de ser vetada pelas autoridades o incentivo à industrialização de carne bovina (Pawlovski \& Schultz, 1972). Atualmente, em locais como Cabo Verde (África) é comum a prática de reutilização de esgoto em culturas experimentais de milho e girassol, (Marecos do Monte et al, 1993). 
COELHO, W.M.; CAMPOS, D.M.B. Avaliação da eficiência de um sistema de lagoas de estabilizaçăo de esgotos na remoção de ovos de helmintos: proposta para a determinação do percentual de recuperação de ovos de helmintos através do método "OMS". Rev. Pat. Trop.25 ( 1 ): 61-72, jan./jun. 1996

Assim, em culturas irrigadas com esgoto sem tratamento não apenas os nematodas mas também os cestodas podem constituir uma fonte de contaminação para os trabalhadores rurais.

No efluente da lagoa primária, 2 das 12 amostras examinadas, ou seja $16,6 \%$, encontraram-se fora dos padrões recomendados pela OMS, ou seja, apresentaram mais que 1 ovo de nematoda por litro. Os percentuais de remoção de ovos de $A$. lumbricoides e ancilostomídeos foram respectivamente, para cada uma das duas amostras, de $91,6 \%$ e $92 \%$ e $95,6 \%$ e $97,5 \%$. Estes resultados foram inferiores aos encontrados por Mara \& Silva (1986). Estes autores registraram percentuais de 99 - 100\% e 98 - 100\% de remoção de ovos de Ascaris lumbricoides e ancilostomídeos, respectivamente, em lagoas facultativas primárias, num tempo de retenção de 18,9 dias.

Conforme Sacramento (1990) para se conseguir um efluente que satisfaça aos critérios da OMS, deve-se ter um tempo de retenção superior a 20 dias. No sistema em questão acredita-se que este tempo não ultrapasse 20 dias.

No efluente final, ou seja, no tratamento secundário todas as 12 amostras examinadas apresentaram valores negativos. Portanto, no período desta amostragem, em termos de critérios parasitológicos a obedecer este efluente poderia ser utilizado em irrigações restritas (de cereais, forragens, pastos e árvores) conforme recomendaçб̃es da OMS (WHO, 1989).

\section{AGRADECIMENTOS}

Ao Laboratório de Análises de Águas Residuárias da Empresa Saneamento de Goiás S/A, onde foram realizados os exames parasitológicos, ao Prof. Doutor Carlos Marcos Batista (Instituto de Ciências Humanas e Letras - UFG) pela contribuição na análise estatística dos dados.

\section{SUMMARY}

Evaluation of the Efficiency of a System of Waste Stabilization Ponds in the Remotion of Helminths Eggs - a Proposal for the Determination of the Retrieval Rate of Helminth Eggs through the Schwartzbrod Method.

This study verified the capacity of retention of helminths eggs in a system composed by waste stabilization ponds, and for that purpose three methods were tested and evaluated (EXTRABES, Leeds $I$ and the A method, recommended by the World Health Organization, described by Schwartzbrod (WHO, 1989).
COELHO, W.M.; CAMPOS, D.M.B. Avaliação da eficiência de um sistema de lagoas de estabilização de esgotos na remoção de ovos de helmintos: proposta para a determinação do percentual de recuperação de ovos de helmintos através do método "OMS". Rev. Pat. Trop.25 ( 1 ): 61-72, jan./jun. 1996

As no data were available on the retrieval of helminths eggs by the Schwartzbrod method, a technique was proposed to determine this percentage. The assays were carried out on samples with sedimentable solid volumes ranging from $1,0 \mathrm{ml}$ to $20,0 \mathrm{ml}$ with a retrieval rate of $18 \%$ and $3,3 \%$, respectively. The number of helminth eggs retrieved through this method was larger in samples with less sedimentable solids. These results show that when carrying out a sewage parasitological examination, the amount of solid sediments must be determined in order to apply the correspondent recuperation rates to the values of solids found in the original sample.

Comparing the obtained results, the Schwartzbrod method was 1,5 fold more efficient in recuperating helminth eggs than the EXTRABES and 7,8 fold than the Leeds I method.

The output of the first lake was not appropriate in relation with the removal of helminth eggs in $16,6 \%$ of the samples for utilization in agriculture, according to the World Health Organization patterns.

All the examined samples in the final output. presented a negative value. It was concluded that for the period studied, in terms of parasitological criteria to be followed, the final output of the system could be used in restricted irrigations, according to the World Health Organization recommendations.

KEYWORDS: Effluents; helminth eggs; reutilize, sewer stabilization lakes

\section{REFERÊNCIAS BIBLIOGRÁFICAS}

APHA. (1989). Standard methods for examination of water and wastewater. 17th ed. Americam Public Health Association. Washington.

Ayres, R.; Lee, D.; Mara, D. (1989). The enumeration of human intestinal nematod eggs in raw and treated wastewaters. Tropical Public Health Engineering. March. Leeds. U.K.

Ayres, R. Stott, R; Lee, D.L.; Mara D. D.; Silva, S. A. (1991). Comparison of techniques for the enumeration of human parasitic helminth eggs in treated wastewater. Environmental Technology, vol. 12 pp. 617-623. Leeds. U.K.

Crispim, W. M. C. (1992). Avaliação do comportamento de um dos sistemas de lagoas de estabilização em série, tratando esgoto domésticos - Região Centro-Oeste do Brasil. V SILUBESA. ABES/APRH Lisboa.

Mara, D. D. \& Silva S. A. (1986). Removal of intestinal nematode eggs in tropical waste stabilization ponds. Journal of Tropical Medicine and Hygiene. 89, pág. 71-74.

Mara, D. D. \& Cairncross, S. (1990). Directrices para el uso sin riesgos de aguas residuales en agricultura y acuicultura. Ginebra. OMS, 212 pp.

Marecos do Monte, M. H.; Crispim, W. M. C.; Costa, A. V. (1993). Protocolo dos ensaios de utilização para rega do efluente da ETAR do Mindelo. Relatório interno do NHS/DH/LNEC. Lisboa. 
COELHÖ, W.M.; CAMPOS, D.M.B. Avalią̧ăo da eficiencia de um sistema de lagoas de estabilizaqło de esgotos na remoção de ovos de helmintos: proposta para a determinaçáo do percentual de recuperação de ovos de helmintos através do método "OMS". Rev. Pat. Trop.25 ( 1 ): 61-72, jan./jun. 1996

Marecos do Monte, M. H. (1993). Wastewater treatment ponds - A low technology for conventional and advanced treatment. Não publicado. Lisboa.

Pawlowski, Z. S. (1984). Intestinal helminthiases. Medicine International, pp. 181-189.

Pawlowski, Z. \& Schultz G. M.(1972). Teniasis and cisticercosis. Advances in Parasitology, 10:301-323.

Sacramento, A. (1990). Contaminação e remoçăo de ovos de helmintas em águas residuais municipais. LNEC/NHS. Portugal.

Siqueira, E. Q. (1996). Aplicaçăo do modelo de qualidade de água (QUAL2E) na modelaçăo do rio Meia Ponte (GO). Dissertação de mestrado. USP, São Carlos.

World Health Organization (1989). Health guidelines for use of wastewater in agriculture and acquaculture. Technical Report Series. 778. WHO, Geneva.

World Health Organization (1991). Intestinal parasites. Basic laboratory methods in medical parasitology. pp. 67-111. Geneva. 